\title{
A New Precoding for Linear Dispersion Codes to Reduce the Spatial Correlation in Transmitter
}

\author{
Qi Wang, and Wen Chen \\ Department of Electronic Engineering \\ Shanghai Jiaotong University \\ Shanghai, P. R. China 200240 \\ Email: qwang@ece.unm.edu and wenchen@sjtu.edu.cn
}

\begin{abstract}
In this paper we study the data-error performance of Linear Dispersion (LD) codes under spatial correlated multiple-input multiple-output (MIMO) channels. We propose a new precoding algorithm for $L D$ codes by minimizing the pairwise error probability (PEP). This precoding can totally remove the spatial correlation existing in the transmitter. Particularly, the precoding process can be integrated into the LD codes design process, which means that there is no extra computation required in the coding process.
\end{abstract}

\section{INTRODUCTION}

Recently, multiple-input multiple-output (MIMO) technology is an active research area in wireless communication. Linear dispersion (LD) codes introduced in [1] is a good candidate for high-data-rate MIMO wireless communication. The codeword of LD codes is a linear combination of certain dispersion matrices over space and time with the transmitted symbols as combining coefficients. The encoding of LD codes is simple. Furthermore, LD codes can be decoded very efficiently by the polynomial-complexity maximum-likelihood (ML) detector, i.e., the sphere decoder.

A common assumption in the design of space-time coding for MIMO wireless communication system is that each channel between the pairs of transmit-receive antennas is spatially independent [1], [2]. However, this is just an ideal situation. In practice, spatial correlation between channels exists, and highly affects the capacity of the MIMO channel as well as the performance of space-time codes (STCs) [3]-[5].

In this paper, we study the pairwise error probability (PEP) of LD codes in MIMO wireless communication system suffered spatial correlation. According to analysis of the pairwise error probability (PEP), we derive a new precoding criterion for LD codes in spatially correlated slow-fading channels to reduce the spatial correlation in transmitter. We assume that no channel knowledge is known at the transmitter and perfect channel knowledge is known at the receiver. We first introduce the system model of MIMO and the basic structure of LD code in Section II, followed by our precoding criterion under spatial correlated channels. In Section III, we present several precoding design examples. In the end of paper, we conclude this paper.

\section{DESIGN CRITERION}

In this section, we will briefly introduce the multiple-input multiple output (MIMO) model and the linear dispersion code
(LD code) at first. Then we will present the design criterion for our proposed precoding.

\section{A. System Model and LD codes}

We consider an $M_{T} \times M_{R}$ MIMO wireless communication system working through a frequency flat-fading channel. The commonly adopted model for this system is [2]

$$
\mathbf{Y}=\sqrt{\frac{E_{s}}{M_{T}}} \mathbf{H} \mathbf{S}+\mathbf{N}
$$

where $E_{s}$ is the total average transmitting energy during one symbol period, $\mathbf{H}$ is the $M_{R} \times M_{T}$ channel transfer function and, $\mathbf{N}$ is the $M_{R} \times T$ zero mean circularly symmetric complex Gaussian noise vector with covariance matrix $N_{0} \mathbf{I}_{M_{R}}, \mathbf{Y}$ and $\mathbf{S}$ are the received codeword and transmitting codeword over symbol period $T$, respectively.

LD codes are good candidates for high-data-rate MIMO wireless communication. Assume that we transmit $K r$-QAM symbols $\left\{c_{i}\right\}_{i=1}^{K}$ with unit average energy over $T$ symbol intervals, the LD transmission codeword is given by [1]

$$
\mathbf{S}=\sum_{k=1}^{K} \mathbf{A}_{k} c_{k}+\sum_{k=1}^{K} \mathbf{B}_{k} c_{k}^{*}
$$

where $\{\mathbf{A}, \mathbf{B}\}_{k=1}^{K}$ are the LD matrices. Thus, the transmission rate of the $\mathrm{LD}$ codes is

$$
R=\frac{K \log _{2} r}{T}
$$

Also the LD matrices $\{\mathbf{A}, \mathbf{B}\}_{k=1}^{K}$ should satisfy the energy constraint expressed by

$$
\sum_{k=1}^{K} \operatorname{Tr}\left(\mathbf{A}_{k} \mathbf{A}_{k}^{H}+\mathbf{B}_{k} \mathbf{B}_{k}^{H}\right)=M_{T} T .
$$

\section{B. Precoding under Spatially Correlated Channels}

Traditional LD codes design is with the assumption that each channel between the transmit antennas and receive antennas is spatially independent. However, due to the limited physical size of antennas and bad transmission conditions under some circumstances, the spatial correlation between each channel exists. Research showed that spatial correlation of channels highly influences the capacity of the MIMO channel as well as the performance of space-time codes (STCs) [3], 
[4]. Therefore, it's very crucial to find out how the spatial correlation affects the capacity of the MIMO channels and the performance of STCs and how to find a new way to reduce such influences.

Assuming that a codeword $\mathbf{S}^{i}$ is transmitted. Given that the receiver constructs an ML estimate of the transmitted codeword based on the perfect channel knowledge, the probability that the receiver mistakenly decodes the transmitted codeword $\mathbf{S}^{i}$ for codeword $\mathbf{S}^{j}$ is called pairwise error probability (PEP) [2], i.e.,

$$
\begin{aligned}
\mathbf{P}\left(\mathbf{S}^{i} \rightarrow \mathbf{S}^{j} \mid \mathbf{H}\right) & =\mathcal{Q}\left(\sqrt{\frac{E_{s}\left\|\mathbf{H}\left(\mathbf{S}^{i}-\mathbf{S}^{j}\right)\right\|_{F}^{2}}{2 M_{T} N_{0}}}\right) \\
& =\mathcal{Q}\left(\sqrt{\frac{\rho\left\|\mathbf{H}\left(\mathbf{S}^{i}-\mathbf{S}^{j}\right)\right\|_{F}^{2}}{2 M_{T}}}\right) .
\end{aligned}
$$

This equation is valid for MIMO channels with and without spatial correlation.

For the MIMO channel with spatial correlation, The channel model is given by [2]

$$
\operatorname{vec}(\mathbf{H})=\mathbf{R}^{\frac{1}{2}} \operatorname{vec}\left(\mathbf{H}_{w}\right),
$$

where $\mathbf{H}_{w}$ is the spatially white $M_{R} \times M_{T}$ MIMO channel without spatial correlation and $\mathbf{R}$ is the $M_{T} M_{R} \times M_{T} M_{R}$ covariance matrix defined as

$$
\mathbf{R}=\mathcal{E}\left[\operatorname{vec}(\mathbf{H}) \operatorname{vec}(\mathbf{H})^{H}\right] .
$$

$\mathbf{R}$ is a positive semi-definite Hermitian matrix. Although the model described above is capable of capturing any correlation effects between the element of $\mathbf{H}$, people often adopt another easy-handling model with a little loss of generality. But this model is adequate in most of the cases. The model is given by [2]

$$
\mathbf{H}=\mathbf{R}_{r}^{\frac{1}{2}} \mathbf{H}_{w} \mathbf{R}_{t}^{\frac{T}{2}},
$$

where $\mathbf{R}_{t}$ is the transmit covariance matrix and $R_{r}$ is the receive covariance matrix. It's easy to verify that $\mathbf{R}, \mathbf{R}_{r}$ and $\mathbf{R}_{t}$ are related by

$$
\mathbf{R}=\mathbf{R}_{t}^{T} \otimes \mathbf{R}_{r},
$$

where $\otimes$ is the tensor product. Under spatially correlated channels, we have

$$
\|\mathbf{H E}\|_{F}^{2}=\operatorname{vec}\left(\tilde{\mathbf{H}}^{H}\right)^{H} \mathbf{R}_{s}^{\frac{1}{2}}\left(\mathbf{I}_{M_{R}} \otimes \mathbf{E E}^{H}\right) \mathbf{R}_{s}^{\frac{H}{2}} \operatorname{vec}\left(\tilde{\mathbf{H}}^{H}\right)
$$

where $\mathbf{E}$ is the differences between two codewords, namely $\left(\mathbf{S}^{i}-\mathbf{S}^{j}\right)$. The moment generating function [6], [7] of the random variable $\|\mathbf{H E}\|_{F}^{2}$ is denoted by $\psi_{\|\mathbf{H E}\|_{F}^{2}}(v)$. Assuming that Rayleigh slow-fading is with $\mathbf{H}=\mathbf{R}_{r}^{\frac{1}{2}} \mathbf{H}_{w} \mathbf{R}_{t}^{\frac{T}{2}}$. By using the moment generating function of $\|\mathbf{H E}\|_{F}^{2}$, the expression of PEP could be simplified to

$$
\begin{aligned}
\mathbf{P}(\mathbf{E} \mid \mathbf{H}) & =\frac{1}{\pi} \int_{0}^{\frac{\pi}{2}}\left|\mathbf{I}_{M_{T} M_{R}}+\frac{\rho \mathbf{R}_{r} \otimes\left(\mathbf{E} \mathbf{E}^{H} \mathbf{R}_{t}\right)}{4 M_{T} \sin ^{2} \theta}\right|^{-1} d \theta \\
& \leq \frac{1}{2 \operatorname{det}\left(\mathbf{I}_{M_{R} M_{T}}+\frac{\rho}{4 M_{T}} \mathbf{R}_{r} \otimes \mathbf{E E}^{H} \mathbf{R}_{t}\right)}
\end{aligned}
$$

Proposition 1. For slow-fading Rayleigh MIMO channels, if there exists spatial correlation in transmitter but guarantees the full rank of matrix $\mathbf{R}_{t}$, such spatial correlation can be reduced by effective precoding of $L D$ codes. The precoding process is formalized by

$$
\begin{aligned}
& \tilde{\mathbf{A}}_{k}=\mathbf{R}_{t}^{-\frac{1}{2}} \mathbf{A}_{k} \quad \text { for } k=1, \ldots, K \\
& \tilde{\mathbf{B}}_{k}=\mathbf{R}_{t}^{-\frac{1}{2}} \mathbf{B}_{k} \quad \text { for } k=1, \ldots, K
\end{aligned}
$$

Proof: In order to prove the proposition, we will use the following two lemmas from matrix analysis theorem [8].

Lemma 1. Let matrices $\mathbf{A}=\left(a_{i j}\right)_{m \times p}, \mathbf{B}=\left(b_{i j}\right)_{l \times q}, \mathbf{C}=$ $\left(c_{i j}\right)_{p \times n}, \mathbf{D}=\left(d_{i j}\right)_{q \times r}$. Then we have

$$
(\mathbf{A} \otimes \mathbf{B})(\mathbf{C} \otimes \mathbf{D}) \quad=\quad \mathbf{A C} \otimes \mathbf{B D}
$$

Lemma 2. For matrix $\mathbf{A}_{m \times n}$ and matrix $\mathbf{B}_{n \times m}$, there always exists an equality

$$
\operatorname{det}\left(\mathbf{I}_{m}+\mathbf{A B}\right)=\operatorname{det}\left(\mathbf{I}_{n}+\mathbf{B A}\right)
$$

$R_{t}$ is guaranteed to be full rank. So it's a positive semidefinite Hermitian matrix, which means that it can be expressed as

$$
\mathbf{R}_{t}=\mathbf{R}_{t}^{\frac{1}{2}} \mathbf{R}_{t}^{\frac{H}{2}}
$$

Thus, (8) can be rewritten as

$$
\mathbf{P}(\mathbf{E} \mid \mathbf{H}) \leq \frac{1}{2 \operatorname{det}\left(\mathbf{I}_{M_{R} M_{T}}+\frac{\rho}{4 M_{T}} \mathbf{R}_{r} \mathbf{I} \otimes \mathbf{E E}^{H} \mathbf{R}_{t}^{\frac{H}{2}} \mathbf{R}_{t}^{\frac{1}{2}}\right)} .
$$

By using the conclusion of Lemma 1 , this inequality can be rewritten as

$$
\mathbf{P}(\mathbf{E} \mid \mathbf{H}) \leq \frac{1}{2 \operatorname{det}\left(\mathbf{I}_{M_{R} M_{T}}+\frac{\rho}{4 M_{T}}\left(\mathbf{R}_{r} \mathbf{E E}^{H} \mathbf{R}_{t}^{\frac{1}{2}}\right) \otimes\left(\mathbf{I R}_{t}^{\frac{H}{2}}\right)\right)} .
$$

By using the equality in Lemma 2, we have

$$
\mathbf{P}(\mathbf{E} \mid \mathbf{H}) \leq \frac{1}{2 \operatorname{det}\left(\mathbf{I}_{M_{R} M_{T}}+\frac{\rho}{4 M_{T}} \mathbf{I R}_{t}^{\frac{H}{2}} \otimes \mathbf{R}_{r} \mathbf{E} \mathbf{E}^{H} \mathbf{R}_{t}^{\frac{1}{2}}\right)}
$$

Again using Lemma 1, we can finally get

$$
\mathbf{P}(\mathbf{E} \mid \mathbf{H}) \leq \frac{1}{2 \operatorname{det}\left(\mathbf{I}_{M_{R} M_{T}}+\frac{\rho}{4 M_{T}} \mathbf{I R}_{r} \otimes \mathbf{R}_{t}^{\frac{1}{2}} \mathbf{E E}^{H} \mathbf{R}_{t}^{\frac{H}{2}}\right)} .
$$

Let $\{\mathbf{A}, \mathbf{B}\}_{k=1}^{K}$ be the linear dispersion matrices designed for MIMO channel without spatial correlation. For the case that spatial correlation exists, assume that we take the precoding process such as

$$
\begin{aligned}
& \tilde{\mathbf{A}}_{k}=\mathbf{R}_{t}^{-\frac{1}{2}} \mathbf{A}_{k}, \quad \text { for } k=1, \ldots, K, \\
& \tilde{\mathbf{B}}_{k}=\mathbf{R}_{t}^{-\frac{1}{2}} \mathbf{B}_{k}, \quad \text { for } k=1, \ldots, K \text {. }
\end{aligned}
$$


Then the codeword after precoding $\tilde{\mathbf{S}}^{i}$ should be $\mathbf{R}^{-\frac{1}{2}} \mathbf{S}^{i}$, which means that in this case the difference between two codewords is $\tilde{\mathbf{E}}=\mathbf{R}^{-\frac{1}{2}} \mathbf{E}$. Plug this into (14), we can get

$$
\begin{aligned}
\mathbf{P}(\mathbf{E} \mid \mathbf{H}) & \leq \frac{1}{2 \operatorname{det}\left(\mathbf{I}_{M_{R} M_{T}}+\frac{\rho}{4 M_{T}} \mathbf{I} \mathbf{R}_{r} \otimes \mathbf{R}_{t}^{\frac{1}{2}} \tilde{\mathbf{E}} \tilde{\mathbf{E}}^{H} \mathbf{R}_{t}^{\frac{H}{2}}\right)} \\
& =\frac{1}{2 \operatorname{det}\left(\mathbf{I}_{M_{R} M_{T}}+\frac{\rho}{4 M_{T}} \mathbf{R}_{r} \otimes \mathbf{R}_{t}^{\frac{1}{2}} \mathbf{R}_{t}^{-\frac{1}{2}} \mathbf{E} \mathbf{E}^{H} \mathbf{R}_{t}^{-\frac{H}{2}} \mathbf{R}_{t}^{\frac{H}{2}}\right)} \\
& =\frac{1}{2 \operatorname{det}\left(\mathbf{I}_{M_{R} M_{T}}+\frac{\rho}{4 M_{T}} \mathbf{I R}_{r} \otimes \mathbf{I E E} \mathbf{E}^{H} \mathbf{I}\right)}
\end{aligned}
$$

According to (17), one can easily find out that the spatial correlation in the transmitter represented by $\mathbf{R}_{t}$ is totally reduced by the precoding process applied to LD codes.

We have several remarks for this precoding design criterion.

Remark 1. This precoding method is valid when the transmit covariance matrix $\mathbf{R}_{t}$ is full rank. Otherwise $\mathbf{R}_{t}$ is a singular matrix. Then we cannot decompose it to the product of $\mathbf{R}_{t}^{\frac{1}{2}}$ and $\mathbf{R}_{t}^{\frac{H}{2}}$.

Remark 2. From the energy point of view, spatial correlation actually reduces the transmit energy. At the meantime, our precoding process indeed increase the transmit energy to combat such spatial correlation in the transmitter. Given a specific transmit covariance matrix, the precoding design criterion tells how to increase the transmit energy in order to totally reduce the spatial correlation at the transmitter.

Remark 3. Usually people can get the transmit covariance matrix from the geometry of transmit antennas. Therefore, when people design an LD code for a given MIMO channel with spatial correlation at the transmitter, they can integrate the precoding processing into the LD code design just by conducting a linear transform to the LD matrices design for independent MIMO channel. There is no extra computation at the coding process.

\section{EXAMPLES OF PRECOdING DESIGN}

In this section we give several precoding design examples. First, We use the cyclotomic LD codes design method proposed by Zhang et al. in [9] to generate the trace-orthonormal full-diversity cyclotomic LD codes for the MIMO channels without spatial correlation. Then we use some typical covariance matrices $\mathbf{R}_{t}$ in transmitter to simulate the spatially correlated channel. According to a certain covariance matrix, we apply the precoding process to the LD codes designed for MIMO channels without correlations. At last we compare the performances of these LD codes under different circumstances. We assume that no spatial correlation exists in receiver.

Example 1. Here we take a $2 \times 2$ MIMO wireless communication system. The time interval of the codeword $T$ is equal to 2 symbol periods. The size of $L D$ matrices is chosen to be the product of $T$ and $M_{T}$. Then we have $\left\{\mathbf{A}_{k}, \mathbf{B}_{k}\right\}_{k=1}^{4}$ to construct the LD codewords.

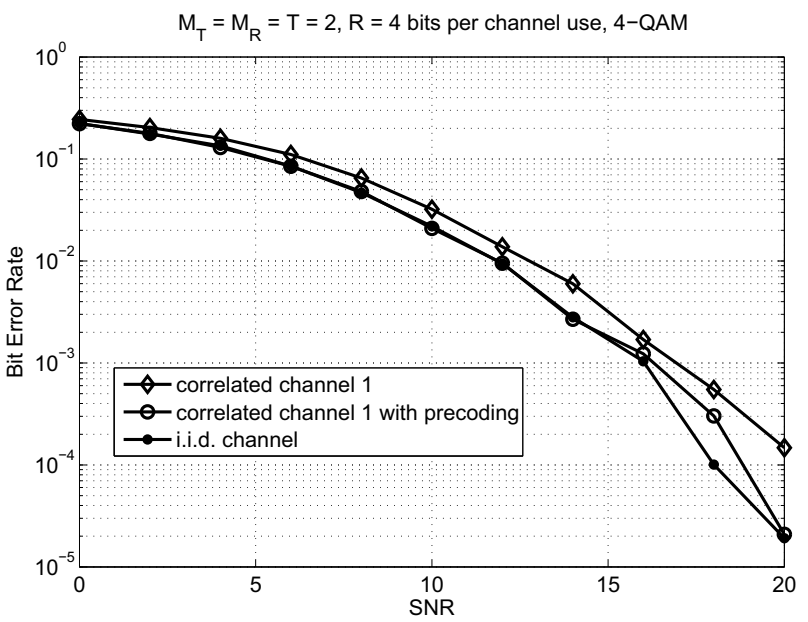

Fig. 1.

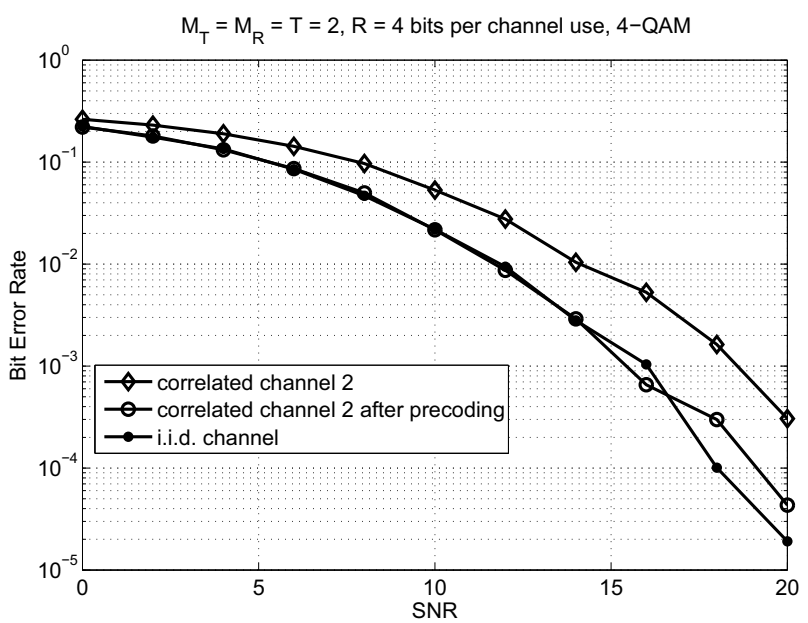

Fig. 2. MIMO

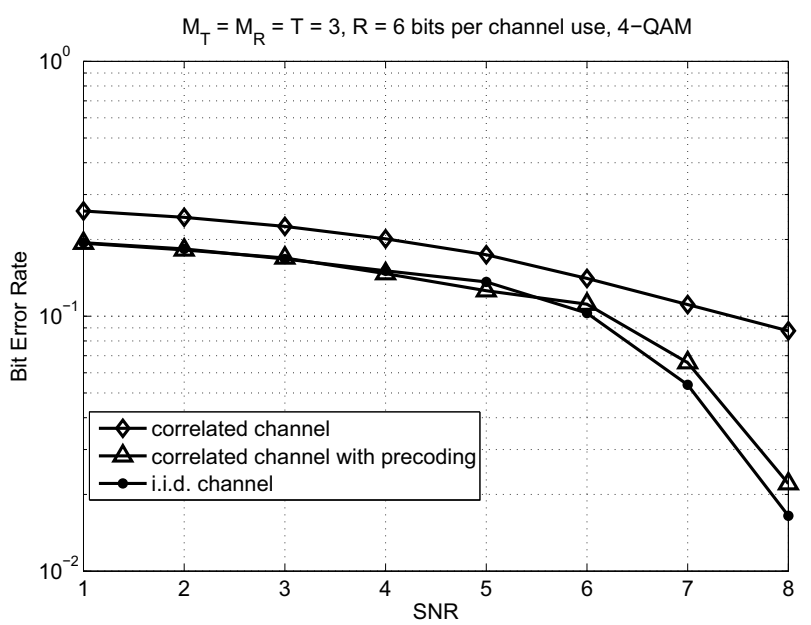

Fig. 3. MIMO 
One typical transmit covariance matrix is

$$
\mathbf{R}_{t}=\left(\begin{array}{cc}
1 & 0.7 e^{j \pi / 4} \\
0.7 e^{-j \pi / 4} & 1
\end{array}\right)
$$

Let $\mathbf{P}$ be the precoding matrix, then

$$
\mathbf{P}=\mathbf{R}_{t}^{-\frac{1}{2}}=\left(\begin{array}{cc}
1.2964-0.0000 i & -0.3743-0.3743 i \\
-0.3743+0.3743 i & 1.2964
\end{array}\right)
$$

Let $\left\{\tilde{\mathbf{A}}_{k}, \tilde{\mathbf{B}}_{k}\right\}_{k=1}^{4}$ denote the LD matrices after precoding. Thus the precoding process could be represented by

$$
\tilde{\mathbf{A}}_{k}=\mathbf{P A}_{k}, \tilde{\mathbf{B}}_{k}=\mathbf{P} \mathbf{B}_{k}
$$

for all $k=1, \ldots, 4$. Fig. 1 gives the performance of the LD codes (with and without precoding) under such spatial correlation. In order to illustrate the effort of precoding, we also give the performance of $L D$ codes without precoding under uncorrelated channels. From this figure, it's self-evident that this simulation result is coherent with our theoretical result.

Another typical transmit covariance matrix of $2 \times 2$ MIMO system is

$$
\mathbf{R}_{t}=\left(\begin{array}{cc}
1 & 0.9 e^{j \pi / 4} \\
0.9 e^{-j \pi / 4} & 1
\end{array}\right)
$$

The corresponding precoding matrix is given by

$$
\mathbf{P}=\left(\begin{array}{cc}
1.9439 & -0.8615-0.8615 i \\
-0.8615+0.8615 i & 1.9439
\end{array}\right)
$$

By using the same precoding procedures, we can get the performance of the LD codes (with and without precoding) under such spatial correlation in Fig. 2. The simulation result is also coherent with our theoretical result from part II.

Example 2. Here we give an example of a $3 \times 3$ MIMO wireless communication system. We choose the time interval of the codeword to be 3 symbol periods. Also the size of $L D$ matrices is chosen to be the product of $T$ and $M_{T}$.

In this case, we use the following transmit covariance matrix:

$$
\mathbf{R}_{t}=\left(\begin{array}{ccc}
1 & 0.9 e^{j \pi / 4} & 0.81 e^{j \pi / 2} \\
0.9 e^{-j \pi / 4} & 1 & 0.9 e^{j \pi / 4} \\
0.81 e^{-j \pi / 2} & 0.9 e^{-j \pi / 4} & 1
\end{array}\right)
$$

The corresponding precoding matrix $\mathbf{P}$ is

$$
\left(\begin{array}{ccc}
2.0055+0.0000 i & -0.7609-0.7609 i & -0.0000-0.2887 i \\
-0.7609+0.7609 i & 2.6852-0.0000 i & -0.7609-0.7609 i \\
-0.0000+0.2887 i & -0.7609+0.7609 i & 2.0055
\end{array}\right)
$$

By using the same precoding procedures as before, we can get the performance of the $L D$ codes (with and without precoding) under such spatial correlation in Figure 3. Due to the sharp increase of computation caused by the enlarged size of MIMO system, we just calculate the bit error rate up to $8 \mathrm{~dB}$. However, the result is still compatible with our theoretical result.

\section{CONCLUSIONS}

In this paper we study the bit-error performance of LD codes communicating in spatially correlated MIMO channels. A new precoding scheme is proposed to reduce the spatial correlation in transmitter. The precoding is applied to nonsingular transmit covariance matrix and requires no extra computation in LD coding process. Three simulation examples is presented to verify our theoretical results.

\section{ACKNOWLEDGMENT}

The authors would like to thank Jun Li for some enlighten discussion.

This work is supported by NSF China \#60972031, \#60672067 and \#60632040, by Cultivation Fund of the Key Scientific and Technical Innovation Project, Ministry of Education of China \#706022, by Program for New Century Excellent Talents in University \#NCET-06-0386, by PUJIANG Rencai \#07PJ4046, and by Huawei funding \#YJCB2008048WL.

\section{REFERENCES}

[1] B. Hassibi and B. M. Hochwald, "High-rate codes that are linear in space and time," IEEE Trans. Inf. Theory, vol. 48, pp. 1804-1823, Jul. 2002.

[2] A. Paulraj, R. Nabar, and D. Gore, Introduction to Space-Time Wireless Communications. Beijing, China: Tsinghua University Press, 2005.

[3] C. Chuah, D. Tse, J.Kahn, and R. Valenzuela, "Capacity scaling in mimo wireless systems under correlated fading," IEEE Trans. Inf. Theory, vol. 48, pp. 637-650, Mar. 2002.

[4] D.-S. Shiu, G. J. Foschini, M. J. Gans, and J. M. Kahn, "Fading correlation and its effect on the capacity of multielement antenna systems," IEEE Trans. Commun., vol. 48, pp. 502-513, Mar. 2000.

[5] B. Clerckx et al., "Design and performance of space-time codes for spatially correlated mimo channels," IEEE Trans. Commun., vol. 55, pp. 64-68, Feb. 2007.

[6] A. Hedayat, H. Shah, and A. Nosratinia, "Space-time signaling in correlated channels," in IEEE Wireless Communications and Networking Conference (WCNC, '05), unkown, unkown, Mar. 2005, pp. 544-549.

[7] G. L. Turin, "The characteristic function of hermitian quadratic forms in complex normal random variables," Biometrika, pp. 199-201, 1960.

[8] R. A. Horn and C. R. Johnson, Matrix Analysis. New York: Cambridge University Press, 1988.

[9] J.-K. Zhang, J. Liu, and K. M. Wong, "Trace-orthonormal full-diversity cyclotomic space-time codes," IEEE Trans. Signal Process., vol. 55, pp. 618-630, 2007. 